\title{
Expressão citofotométrica quantitativa da CASPASE-3 e do CD-34 no carcinoma ductal infiltrante de mama
}

\author{
Cytophotometric expressions of CASPASE-3 and CD-34 in breast cancer
}

Fábio Santana dos Passos ${ }^{1}$; Ronaldo Máfia Cuenca, TCBC-DF²; Osvaldo Malafaia, ECBC-PR²; Jurandir Marcondes Ribas-Filho, TCBC-PR²; Nicolau Gregori Czeczko,TCBC-PR²; Paulo Afonso Nunes Nassif, TCBC-PR²; Carmen Austrália Paredes Marcondes Ribas²

\section{RE S U M O}

\begin{abstract}
Objetivo: Descrever, correlacionar e comparar a expressão dos marcadores tumorais CD-34 (angiogênese) e caspase-3 (apoptose) em carcinoma ductal invasor de mama. Métodos: Foram utilizados 22 casos de adenocarcinoma infiltrante de mama provenientes de blocos de parafina e, após preparo específico para imunoistoquímica, 15 apresentaram leitura satisfatória e foram avaliados pelo sistema de fotocitometria de imagem SAMBA $4000 ®$ e software IMMUNO®. Os parâmetros analisados foram o índice de marcagem e densidade óptica. Resultados: Para o CD-34 não houve normalidade dos dados na análise do índice de marcagem, com obtenção de $P=0,019$, havendo normalidade para a análise da densidade óptica, com $P=0,199$. Para a caspase-3 houve normalidade de dados para o índice marcagem com $P=0,306$ e para a densidade óptica com $P=0,114$; não houve diferença estatística significativa entre eles em relação à média do índice de marcagem $(P=0,872)$ e da densidade óptica $(P=0,816)$, quando analisados os parâmetros que definem a expressão dos marcadores; existiu tendência à associação entre a densidade óptica e o índice de marcagem do marcador tumoral caspase-3, com $P=0,025$. Não foi observada tendência à associação quando comparados densidade óptica e índice de marcagem do marcador tumoral CD-34; índice de marcagem do marcador tumoral caspase-3 e índice de marcagem do marcador tumoral CD-34; e densidade óptica da caspase-3 com a do CD-34. Conclusão: Dos 22 casos incluídos foi possível verificar a expressão do marcador CD-34 em 18 lâminas e da caspase-3 em 22 lâminas; Para o CD-34 não houve normalidade dos dados na análise do índice de marcagem, havendo sim normalidade para a análise da densidade óptica. Para a caspase-3 houve normalidade de dados tanto para o índice de marcagem como para a densidade óptica. Existe tendência à associação entre a densidade óptica e o índice de marcagem da caspase-3. Não foi observada tendência quando comparados densidade óptica e índice de marcagem no CD-34.
\end{abstract}

Descritores: Neoplasias da Mama. Apoptose. Imunoistoquímica. Citometria por imagem.

\section{INTRODUÇÃO}

$\mathrm{O}$ câncer constitui o maior problema de saúde pública nos EUA e países desenvolvidos. Estudos estatísticos estimam uma em cada quatro mortes devido a neoplasias malignas. Dentre as mulheres, o da mama figura entre os mais incidentes e com maiores índices de letalidade.

À luz dos atuais conhecimentos não existem dados consistentes que apontem fatores de proteção primária para o câncer de mama, mas sim de redução de risco'. Dentre eles estão a idade acima de 50 anos, local de nascimento (América do Norte e Europa) e histórico de câncer de mama em mãe e irmã².

Quanto ao diagnóstico precoce e métodos de rastreamento populacional, a Sociedade Americana de Câncer recomenda exame clínico anual a partir do 20 anos e mamografias a cada ano a partir dos 40 anos. Entretanto o aumento substancial dos exames mamográficos na década de 80 não afetou os índices de mortalidade nos anos seguintes ${ }^{3}$.
O diagnóstico precoce, então, não só tem impacto nos índices de mortalidade, mas também nas políticas de saúde, alterando as proporções de gastos no tratamento ${ }^{4}$.

Dentre os fatores prognósticos encontram-se o tamanho do tumor, o comprometimento linfonodal, o grau histológico e a idade 5 .

A sobrevida em cinco anos, englobando todos os estágios, atinge $98 \%$, reduzindo-se para $26 \%$ nos casos diagnosticados com metástase à distância .

O desenvolvimento tecnológico permitiu avanços científicos na área de biologia molecular e genética, possibilitando compreender melhor os processos de proliferação celular e fatores ligados à oncogênese. Dentre os envolvidos no processo de desenvolvimento de tumores encontram-se a apoptose e a angiogênese.

O processo de apoptose está intimamente ligado à ação de proteases, denominadas caspases. Dentre estas, a caspase-3 tem sido largamente usada como marcador imunoistoquímico por representar a confluência entre os mecanismos intrínsecos e extrínsecos da apoptose?

Trabalho realizado na Faculdades Integradas da União Educacional do Planalto Central - FACIPLAC, Brasília, Distrito Federal, DF, Brasil. 1. Mestre do Programa de Pós-Graduação em Princípios da Cirurgia do Hospital Universitário Evangélico de Curitiba/Faculdade Evangélica do Paraná, Curitiba, PR-BR. 2. Doutor, Professor Permanente do Programa de Pós-graduação em Princípios da Cirurgia do Hospital Universitário Evangélico de Curitiba/Faculdade Evangélica do Paraná, Curitiba, PR-BR. 
Em relação ao processo de angiogênese, diversos marcadores são utilizados, com o objetivo de expressar a microdensidade vascular ${ }^{8}$.

A análise da expressão celular destes marcadores pode ser executada de diversas maneiras, dentre elas a marcação com técnica de imunoistoquímica analisada subjetivamente pelo patologista ou auxiliado por interpretação computacional ${ }^{9}$.

Existem diversos métodos de análise citofotométrica, dentre eles o SAMBA (Systeme d'analyse microscopique a balayage automatique - Sistema de análise microscópica de busca automática) desenvolvido pela Alcatel (Grenoble - França).

Os objetivos deste estudo no carcinoma ductal infiltrante de mama são: 1 . descrever a expressão dos marcadores tumorais CD-34 e caspase-3; 2.Verificar se havia normalidade dos dados entre eles; 3 . Comparar as expressões em relação ao índice de marcagem e densidade óptica média.

\section{MÉTODOS}

Este trabalho foi avaliado e aprovado pelo Comitê de Ética em Pesquisa da Sociedade Evangélica Beneficente, Curitiba PR.

Foram selecionados 22 casos de operações realizadas por carcinoma ductal invasor com gradações histológicas variadas, obtidos no arquivo do Laboratório Citolab, em Curitiba, PR, Brasil.

Diante do exame histológico realizado por patologista, foram utilizados os blocos de parafina tendo como critério o bom estado de conservação e áreas com boa representatividade do tecido tumoral (análise em microscopia óptica de lâminas coradas pela hematoxilina-eosina).

As lâminas, então, foram preparadas com técnica específica para imunoistoquímica para os marcadores caspase-3 e CD-34 e hematoxilina-eosina.

Para determinação imunoistoquímica de apoptose foi utilizado o anticorpo anti-CPP32 humano, policlonal derivado de coelho, fabricado pela Biogen ${ }$, DakoCytomation $\circledast$, com diluição de 1 microlitro do anticorpo para cada 500 microlitros de diluente.

Para determinação imunoistoquímica do processo de angiogênese utilizou-se o anti-CD-34, anticorpo antihumano, monoclonal derivado de plasma de rato, clone QBEnd/10, tipo IgG, fabricado pela Labdivision $囚$, com a diluição de 1 microlitro para cada 200 microlitros de diluente.

Para quantificação computadorizada utilizou-se o sistema SAMBA 4000®. Esse sistema é constituído de hardware composto por: computador, dois monitores, impressora, microscópio e câmera colorida.

A captura de imagens pelo sistema padroniza as cores verde, azul e vermelha (sistema RGB - "red, green, blue") proporcionando captação homogênea.

A imagem captada é transformada em pontos de imagem (pixel) com área equivalente a $0,16 \mathrm{im}^{2}$ por ponto e enviada ao computador.
O software utilizado para interpretação e análise das imagens foi o IMMUNO 4.0®.

A etapa inicial foi a calibração do sistema, seguindo protocolo específico em 3 fases: controle de entrada de luz; white balance e black balance; calibragem no primeiro campo a ser estudado.

As imagens analógicas tais quais percebidas pelo patologista, foram captadas através da câmara e transformadas em imagens numéricas. Este processo, chamado de numerização da imagem, envolve inicialmente a geração de imagem da matriz em níveis de cinza, e considera 0 como igual a pontos de imagem sem densidade (transparentes) e 255 à maior densidade. Entre os dois níveis de cor, existe gradação em cinza representando a maior ou menor dificuldade de passagem da luz pelo tecido existente na lâmina, ou seja, onde não existe tecido é 0 e quando o tecido é o maior em intensidade, dificultando ao máximo a passagem do luz, é 255. A variação numérica irá representar a diferente concentração do marcador.

Para melhor aproveitamento das imagens em cada lâmina utilizou-se os locais de maior ação do marcador, denominados hot spots. $\mathrm{O}$ aumento utilizado foi de 20 vezes em todo tecido marcado.

$\mathrm{Na}$ análise computacional, o software determina o índice de marcagem, que demonstra o quanto da superfície da lâmina foi marcado (em análise percentual), e a densidade óptica, que demonstra a concentração do marcador na área analisada

Para apresentação da frequência do índice de marcagem do CD-34, as lâminas foram divididas em amostras com índices em cinco grupos de: $30,01 \%$ a $40 \%$; $40,01 \%$ a $50 \% ; 50,01 \%$ a $60 \% ; 60,01 \%$ a 70 e $70,01 \%$ a $80 \%$.

Para a frequência da densidade óptica do CD34, as lâminas foram divididas em amostras com densidade em seis grupos de: 30,01 a 40; 40,01 a 50; 50,01 a 60; 60,01 a 70; 70,01 a 80 e 80,01 a 90.

Para a frequência do índice de marcagem da caspase-3, as lâminas foram divididas em amostras em cinco grupos com índices de: até 20\%; 20,01 a 40\%; 40,01 a $60 \% ; 60,01$ a $80 \%$ e 80,01 a $100 \%$.

Para a distribuição da frequência da densidade óptica da caspase-3, as lâminas foram divididas em sete grupos com densidade de: 20,00 a $30 ; 30,01$ a 40; 40,01 a 50; 50,01 a 60; 60,01 a 70; 70,01 a 80 e 80,01 a 90 .

Os resultados obtidos no estudo foram expressos por médias, medianas, valores mínimos, valores máximos e desvios-padrão.

A condição de normalidade das variáveis quantitativas foi investigada usando-se o teste de Shapiro-Wilks. Para avaliar o grau de associação entre a expressão dos marcadores, foi estimado o coeficiente de correlação de Pearson ou o de Spearman. A comparação dos marcadores foi feita usando-se o teste t de Student para amostras pareadas. Valores de $P<0,05$ indicaram significância estatística. 


\section{RESULTADOS}

Neste estudo foram considerados 22 casos. Com o marcador CD-34 foi possível a leitura de 18 lâminas e com a caspase-3 foram lidas 22 lâminas.

Após a leitura foi elaborada tabela que demonstrou o número de campos lidos, o número de cada caso, o índice de marcagem e a densidade óptica (Tabela 1).

Quando comparadas as expressões dos dois marcadores entre eles, utilizou-se 15 casos, o que corresponde às lâminas lidas pelos dois marcadores (Tabela 2).

\section{Marcador CD-34}

Distribuição de frequência do índice de marcagem Na tabela 3 são apresentadas as distribuições de frequências do índice de marcagem segmentados nos cinco grupos.

Testou-se a hipótese nula de que os dados teem distribuição normal versus a alternativa de que eles não a teem. Os resultados indicaram que não há normalidade dos dados para o índice de marcagem $(P=0,019)$.

Distribuição da frequência da densidade óptica Na tabela 3 são apresentadas as distribuições de frequência da densidade óptica do marcador CD-34, segmentados nos seis grupos.

Testou-se a hipótese nula de que os dados teem distribuição normal versus a alternativa de que eles não a teem. Os resultados dos testes indicaram que, para CD-34, não houve rejeição da hipótese de normalidade dos dados para a densidade óptica $(P=0,199)$.

\section{Marcador caspase-3}

Distribuição de frequência do índice de marcagem $\mathrm{Na}$ tabela 4 são apresentadas as distribuições de frequências do índice de marcagem da caspase-3, segmentados nos cinco gupos.

Tabela 1 - Resultados da análise do índice de marcagem e densidade óptica dos marcadores tumorais CD-34 e caspase-3.

\begin{tabular}{|c|c|c|c|c|c|}
\hline \multirow[t]{2}{*}{$\begin{array}{l}\mathrm{N}^{\circ} \text { de campos lidos } \\
(\mathrm{CD}-34 \text { / caspase- } 3)\end{array}$} & \multirow{2}{*}{$\begin{array}{l}\text { Código } \\
\text { do caso }\end{array}$} & \multicolumn{2}{|c|}{$C D-34$} & \multicolumn{2}{|c|}{ caspase-3 } \\
\hline & & Indice de marcagem & Densidade óptica & Indice de marcagem & Densidade óptica \\
\hline \multirow[t]{2}{*}{$10 / 10$} & K51 & $72,94 \%$ & 64,57 & $70,42 \%$ & 64,28 \\
\hline & K52 & & & & \\
\hline $10 / 10$ & K53 & $67,83 \%$ & 70,64 & $76,95 \%$ & 60,33 \\
\hline \multirow[t]{2}{*}{$10 / 10$} & K54 & $71,51 \%$ & 68,55 & $69,46 \%$ & 62,17 \\
\hline & K55 & & & & \\
\hline $10 / 10$ & K56 & $77,10 \%$ & 49,89 & $67,60 \%$ & 89,7 \\
\hline $10 / 10$ & K57 & $73,47 \%$ & 83,79 & $92,26 \%$ & 78,74 \\
\hline \multirow[t]{2}{*}{$10 / 10$} & K58 & $53,96 \%$ & 79,6 & $82,79 \%$ & 66,73 \\
\hline & K59 & & & & \\
\hline $10 / 10$ & K60 & $75,35 \%$ & 68,86 & $58,26 \%$ & 56,83 \\
\hline $10 / 10$ & K61 & $64,26 \%$ & 79,57 & $57,90 \%$ & 58,73 \\
\hline $10 / 10$ & K62 & $62,77 \%$ & 46,95 & $40,18 \%$ & 59,59 \\
\hline \multirow[t]{3}{*}{$10 / 10$} & K63 & $74,70 \%$ & 62,33 & $76,09 \%$ & 45,2 \\
\hline & K64 & & & & \\
\hline & K65 & & & & \\
\hline \multirow[t]{2}{*}{$10 / 10$} & K66 & $75,04 \%$ & 68,56 & $49,62 \%$ & 55,28 \\
\hline & K67 & & & & \\
\hline \multirow[t]{2}{*}{$10 / 10$} & K68 & $78,92 \%$ & 68,25 & $14,56 \%$ & 27,62 \\
\hline & K69 & & & & \\
\hline $10 / 10$ & K70 & $39,19 \%$ & 30,93 & $48,63 \%$ & 55,57 \\
\hline $10 / 10$ & K71 & $44,09 \%$ & 38,42 & $83,71 \%$ & 49,16 \\
\hline $10 / 10$ & $\mathrm{~K} 72$ & $47,07 \%$ & 41,08 & $73,34 \%$ & 72,38 \\
\hline
\end{tabular}

Tabela 2 - Valores de média, mediana, máximo, mínimo e desvio-padrão do índice de marcagem e densidade óptica dos marcadores CD-34 e caspase-3.

\begin{tabular}{lcccccc}
\hline Variável & $\mathbf{n}$ & Média & Mediana & Mínimo & Máximo & Desvio-padrão \\
\hline Índice de marcagem do CD-34 & 15 & 65,21 & 71,51 & 39,19 & 78,92 & 13,04 \\
Densidade óptica do CD-34 & 15 & 61,47 & 68,25 & 30,93 & 83,79 & 16,20 \\
Índice de marcagem da caspase-3 & 15 & 64,12 & 69,46 & 14,56 & 92,26 & 19,91 \\
Densidade óptica da caspase-3 & 15 & 60,15 & 59,59 & 27,62 & 89,70 & 14,38 \\
\hline
\end{tabular}


Tabela 3 - Distribuição de frequência do índice de marcagem e distribuição de frequência da densidade óptica do marcador CD-34.

\begin{tabular}{lcc}
\hline Indice de marcagem do CD-34 & Frequência & Percentual \\
\hline 30,01 a 40 & 1 & 6,67 \\
40,01 a 50 & 2 & 13,33 \\
50,01 a 60 & 1 & 6,67 \\
60,01 a 70 & 3 & 20,00 \\
70,01 a 80 & 8 & 53,33 \\
Total & 15 & 100 \\
\hline Condição de normalidade: $P=0,019$ (Teste de Shapiro-Wilks) & \\
Densidade óptica & Frequência & Percentual \\
\hline 30,01 a 40 & 2 & 13,33 \\
40,01 a 50 & 3 & 20,00 \\
50,01 a 60 & 0 & 0,00 \\
60,01 a 70 & 6 & 40,00 \\
70,01 a 80 & 3 & 20,00 \\
80,01 a 90 & 1 & 6,67 \\
Total & 15 & 100 \\
\hline
\end{tabular}

Condição de normalidade: $P=0,199$ (Teste de Shapiro-Wilks).

Tabela 4 - Distribuição de frequência do índice de marcagem e distribuição de frequência da densidade óptica do marcador tumoral caspase-3.

\begin{tabular}{lcc}
\hline Indice de marcagem & Frequência & Percentual \\
\hline Até 20 & 1 & 6,67 \\
20,01 a 40 & 0 & 0 \\
40,01 a 60 & 5 & 33,33 \\
60,01 a 80 & 6 & 40,00 \\
80,01 a 100 & 3 & 20,00 \\
Total & 15 & 100 \\
\hline Condição de normalidade: $P=0,306$ (Teste de Shapiro-Wilks) & \\
Densidade óptica & Frequência & Percentual \\
\hline 20,00 a 30 & 1 & 6,67 \\
30,01 a 40 & 0 & 0 \\
40,01 a 50 & 2 & 13,33 \\
50,01 a 60 & 5 & 33,33 \\
60,01 a 70 & 4 & 26,67 \\
70,01 a 80 & 2 & 13,33 \\
80,01 a 90 & 1 & 6,67 \\
Total & 15 & 100 \\
\hline
\end{tabular}

Condição de normalidade: P=0,704 (Teste de Shapiro-Wilks).

Testou-se a hipótese nula de que os dados teem distribuição normal versus a alternativa de que eles não a teem. Os resultados indicaram que não houve rejeição da hipótese de normalidade dos dados para o índice de marcagem $(P=0,306)$.

\section{óptica}

\section{Distribuição de frequência da densidade}

Na tabela 4 são também apresentadas as distribuições de frequências da densidade óptica segmentadas nos sete gupos.
Testou-se a hipótese nula de que os dados teem distribuição normal versus a alternativa de que eles não a teem. Os resultados indicaram que não houve rejeição da hipótese de normalidade dos dados para a densidade óptica ( $P=0,704$ - teste de Shapiro-Wilks).

\section{Correlação entre as expressões dos marcadores CD-34 e caspase-3 \\ Índice de marcagem \\ Testou-se a hipótese nula de que as médias do índice de marcagem do CD-34 e do índice de marcagem}


da caspase-3 são iguais versus a alternativa de que as médias são diferentes.

Na tabela 5 são apresentados os valores de média, mediana, mínimo, máximo e desvio-padrão do índice de marcagem dos dois marcadores e também da diferença entre eles.

$O$ resultado do teste indicou a não rejeição da hipótese nula $(P=0,872)$. Sendo assim, não se pode afirmar que existe diferença significativa entre CD-34 e caspase-3 em relação à média do índice de marcagem.

\section{Densidade óptica}

Testou-se a hipótese nula de que as médias da densidade óptica do marcador CD-34 e a caspase-3 são iguais versus a alternativa de que elas são diferentes.

Na tabela 5 são apresentados os valores de média, mediana, mínimo, máximo e desvio-padrão do índice de marcagem dos dois marcadores e também da diferença entre eles.

O resultado do teste indicou a não rejeição da hipótese nula $(P=0,816)$. Sendo assim, não se pode afirmar que existe diferença significativa entre CD-34 e caspase-3 em relação à média da densidade óptica.

\section{marcadores}

Comparação entre as expressões dos

Para avaliar o grau de associação entre as expressões dos marcadores, foram estimados os coeficientes de correlação.

Em cada situação testou-se a hipótese nula de ausência de correlação entre as expressões dos marcadores versus a alternativa de existência (Tabela 6).
Os resultados dos testes estatísticos indicaram que, para o marcador caspase-3, valores baixos do índice de marcagem correspondem a valores baixos da densidade óptica, bem como valores altos de índice de marcagem correspondem a valores altos de índice de densidade óptica, indicando correlação significativa entre o índice de marcagem e a densidade óptica $(P=0,025)$.

\section{DISCUSSÃO}

O avanço científico e tecnológico das ciências médicas propiciou ao longo dos últimos séculos revoluções em termos de expectativa e qualidade de vida.

Com aumento da expectativa, o câncer vem se configurando como problema de saúde pública em caráter mundial, desafiando a ciência na busca de entender meIhor os processos biológicos envolvidos na gênese desse grupo de moléstias, assim como fatores que possam determinar a prevenção primária e prognóstico.

Pela alta incidência do câncer de mama, a obtenção de amostra para este estudo não apresentou maiores dificuldades. Entretanto o primeiro lote de 50 casos não forneceu cortes histológicos adequados para estudo imunoistoquímico. Esta primeira amostra foi proveniente de operações realizadas entre 2000 e 2004. Um segundo lote com 22 casos foi proveniente de outras realizadas em 2006. Dos 22 blocos selecionados, 15 apresentaram leitura satisfatória pelo sistema SAMBA $4000 \AA$, apresentando, portanto, índice de aproveitamento dos blocos de $68,2 \%$.

Tabela 5 - Valores de média, mediana, valores mínimos, valores máximos, desvio- padrão e valor de $P$ do índice de marcagem dos marcadores e da densidade óptica dos marcadores CD-34 e caspase-3 e da diferença entre ambos.

\begin{tabular}{lccccccc}
\hline & $\mathrm{n}$ & Média & Mediana & Mínimo & Máximo & Desvio-padrão Valor de $\boldsymbol{P}$ * \\
\hline Índice de marcagem do CD-34 & 15 & 65,21 & 71,51 & 39,19 & 78,92 & 13,04 & \\
Índice de marcagem do caspase-3 & 15 & 64,12 & 69,46 & 14,56 & 92,26 & 19,91 & \\
Diferença (caspase3/CD-34) & 15 & $-1,10$ & $-2,05$ & $-64,36$ & 39,62 & 25,82 & 0,872 \\
\hline
\end{tabular}

(*) Teste $t$ de Student para amostras independentes.

\begin{tabular}{lccccccc}
\hline & $\mathrm{n}$ & Média & Mediana & Mínimo & Máximo & Desvio-padrão Valor de $\boldsymbol{P}$ * \\
\hline Densidade óptica do CD 34 & 15 & 61,47 & 68,25 & 30,93 & 83,79 & 16,20 & \\
Densidade óptica da caspase-3 & 15 & 60,15 & 59,59 & 27,62 & 89,70 & 14,38 & \\
Diferença (caspase3/CD-34) & 15 & $-1,31$ & $-6,38$ & $-40,63$ & 39,81 & 21,48 & 0,816 \\
\hline
\end{tabular}

(*) Teste $t$ de Student para amostras independentes.

Tabela 6 - Coeficientes de correlação entre as expressões dos marcadores CD-34 e caspase-3.

\begin{tabular}{lccc}
\hline Variáveis analisadas & $\mathrm{n}$ & $\begin{array}{c}\text { Coeficiente } \\
\text { de correlação }\end{array}$ & Valor de $\boldsymbol{P}$ \\
\hline Índice de marcagem do CD-34 x densidade óptica do CD-34 & 15 & 0,343 & $0,211^{*}$ \\
Índice de marcagem da caspase-3 x densidade óptica da caspase-3 & 15 & 0,574 & $0,025^{* *}$ \\
Índice de marcagem do CD-34 x índice de marcagem da caspase-3 & 15 & $-0,243$ & $0,383^{*}$ \\
Densidade óptica do CD-34 x densidade óptica da caspase-3 & 15 & 0,017 & $0,953^{* *}$ \\
\hline
\end{tabular}

* Coeficiente de correlação de Spearman.

* Coeficiente de correlação de Pearson. 
Este índice sugere que as condições e tempo de armazenagem dos blocos de parafina podem influenciar no aproveitamento para estudo imunoistoquímico.

Para determinação imunoistoquímica do processo de angiogênese utilizou-se o anti-CD-34, anticorpo anti-humano, monoclonal derivado de plasma de rato, clone QBEnd/10, tipo IgG que é de escolha para protocolos que utilizem tecidos em blocos de parafina devido a resistência à desnaturação.

O CD-34 é um marcador pan-endotelial, assim como o CD-31 e o Fator VIII. Entretanto, novos trabalhos científicos que tentam determinar marcadores mais específicos os utilizam como parâmetros para comparação ${ }^{10}$.

O progresso tem avançado na identificação de moléculas que compõe o mecanismo de angiogênese e no desenvolvimento de tratamentos que nela atuem ${ }^{11}$.

A quimioterapia clássica é dependente da perfusão do tumor para distribuição da droga e baixa perfusão tumoral é uma das razões principais para pouca biodisponibilidade da maioria dos quimioterápicos no sítio tumoral. O mesmo raciocínio serviria para radioterapia, pois a terapia anti-angiogênica promove hipóxia, e esta, por sua vez, é uma das principais razões para resistência à radiação, entretanto, existe evidência clínica que a combinação de terapia anti-angiogênica e radioterapia podem funcionar de forma sinérgica ${ }^{12}$.

A caspase-3 tem sido um dos marcadores tumorais mais estudados na pesquisa dos mecanismos apoptóticos pela sua localização na cascata das proteases, representando tanto a via intrínseca como a via extrínseca?

O SAMBA mostrou correlação positiva entre o índice de marcagem e a densidade óptica para a caspase3, sugerindo que a área de marcação acompanhou a intensidade de marcação. Através destes dados pode-se sugerir uniformidade por toda a extensão do comportamento celular no que tange o processo de apoptose.

Estudos imunoistoquímicos sobre a apoptose, através da caspase-3, apontam para a maior expressão em tecidos tumorais que em não tumorais ${ }^{8,13}$.

No presente estudo, os resultados não sugeriram tendência à associação da expressão do CD-34 e da caspase3, não podendo estabelecer, portanto, correlação dos pro- cessos de apoptose e angiogênese, sendo necessários mais trabalhos que estudem diretamente estes dois processos. Em 73\%, as lâminas analisadas apresentaram índice de marcagem acima de 60,01\%, sugerindo resultado semeIhante aos encontrados por outros autores ${ }^{14}$, mesmo com utilização de metodologia diferente. Outro resultado comparável à literatura foi a expressão do marcador CD-34 através de sua densidade óptica ${ }^{15}$.

Um fator que poderia modificar a análise da marcação imunoistoquímica seria o sítio do tumor. No presente estudo foram contemplados apenas tumores provenientes de seu sítio primário, sugerindo novos estudos com mesma metodologia, comparando a expressão do marcador em tumores primários e em sítios metastáticos. Estudo experimental já demonstrou diferença no processo angiogênico da mesma linhagem tumoral dependente do local do tumor (sítio primário x sítio metastático) ${ }^{16}$.

A escolha do SAMBA® foi influenciada por numerosos fatores, sendo preponderante a possibilidade de comparação de resultados, por se tratar de análise com resultados numéricos, e a fácil reprodutibilidade da técnica.

Quando analisado juntamente com a citometria de fluxo, a de imagem produz resultados comparáveis, como descrito por Chen, et al. ${ }^{17}$, em estudo para análise de DNA em câncer de mama.

No presente ensaio não foi observada tendência à correlação quando comparados entre si a densidade óptica e o índice de marcagem dos dois marcadores, portanto nenhuma correlação direta foi estabelecida entre os processos de angiogênese e apoptose.

Dos 22 casos incluídos foi possível verificar a expressão do marcador CD-34 em 18 lâminas e da caspase-3 em 22 lâminas. Para o CD-34 não houve normalidade dos dados na análise do índice de marcagem, havendo sim normalidade para a análise da densidade óptica. Para a caspase-3 houve normalidade de dados tanto para o índice de marcagem como para a densidade óptica. Existe tendência à associação entre a densidade óptica e o índice de marcagem da caspase-3. Não foi observada tendência quando comparados densidade óptica e índice de marcagem no CD-34.

\section{A B S T T R A C T}

Objective: Describe, correlate and compare the expression of the tumor markers CD 34 (angiogenesis) and caspase-3 (apoptosis) in invasive breast adenocarcinoma, through image cytometry with the system SAMBA4000®. Methods: Twenty-two cases of invasive breast adenocarcinoma from paraffin-embedded archival tissue, and after specific prepare, fifteen cases presented a satisfactory lecture with SAM$B A 4000 \circledast$ and could, finally, be evaluated by the software IMMUNO $(n=15)$. The parameters analysed were the label index - in percentage, indicating the marked surface - and the optical density, in pixels - indicator of the marker intensity. The results were tabulated and expressed in averages, mediums, minimum and maximum values. The statistic analysis was realized by the Shapiro-Wilkins, Student test, Pearson's and Spearman's correlation, with statistic significance for values from $p<0,05$. Results: There was no data normality for the label index $\operatorname{CD} 34(p=0,019)$, there was normality in the analysis of the optical density of both markers and label index of the marker Caspase-3. There was no difference relating to the average of the index marker and the optical density when they were compared. Conclusions: There was a tendency to correlate the label index and the optical density of the tumor marker caspase-3, the same did not occur with the tumor marker CD 34. Other analysis did not show any correlation between the two studied markers. Other studies involving theses two cell processes are needed to extend the knowledge of the cancer biomolecular mechanic and to permit new diagnostic and therapeutic strategies.

Key words: Breast Neoplasms. Apoptosis. Immunohistochemistry. Image cytometry. 


\section{REFERENCIAS}

1. Vogel VG. Breast cancer prevention: a review of current evidence. CA Cancer J Clin. 2000; 50(3):156-70

2. Kelsey JL, Gammon MD. The epidemiology of breast cancer. CA Cancer J Clin. 1991; 41(3):146-65.

3. Mettlin C, Smart CR. Breast cancer detection guidelines for women aged 40 to 49 years: rationale for the American Cancer Society reaffirmation of recommendations. CA Cancer J Clin. 1994; 44(4):248-55

4. Max W. The cost of breast cancer in California. Breast Cancer Res [Internet]. 2003 [cited 2007 Mar 04]; [about 1 p.]. Availbale from: www.cbcrp.org

5. Moraes AB, Zanini RR, Turchiello MS, Riboldi J, Medeiros LR. Estudo da sobrevida de pacientes com câncer de mama atendidas no hospital da Universidade Federal de Santa Maria, Rio Grande do Sul, Brasil. Cad Saúde Pública. 2006; 22(10):2219-28.

6. Jemal A, Siegel R, Ward E, Murray T, Xu J, Smigal C, Thun MJ. Cancer Statistics, 2006. CA Cancer J Clin. 2006; 56(2):106-30.

7. Nakopoulou L, Alexandrou P, Stefanaki K, Panayotopoulou E, Lazaris A, Davaris P. Immunohistochemical expression of caspase-3 as na adverse indicator of the clinical outcome in human breast cancer. Pathobiology. 2001; 69(5):266-73.

8. Uzzan B, Nicolas P, Cucherat M, Perret GY. Microvessel density as a prognostic factor in women with breast cancer: a systematic review of the literature and meta-analysis. Cancer Res. 2004 ;64(9):2941-55.

9. Cohen C. Image cytometric analysis in pathology. Hum Pathol. 1996; 27(5):482-94

10. Kumar S, Ghellal A, Li C, Byrne G, Haboubi N, Wang JM, Bundred N. Breast carcinoma: vascular density determined using CD105 antibody correlates with tumor prognosis. Cancer Res. 1999; 59(4):856-61.

11. Gupta K, Zhang J. Angiogenesis: a curse or cure? Postgrad Med J. 2005: 81:236-42.

12. Augustin HG. Translating angiogenesis research into the clinic: the challenges ahead. Br J Radiol. 2003; 76 Suppl 1:S3-10.
13. Tajra JBM, Malafaia O, Czeczko NG, Santos EAA, Ribas CAPM, Wagaa-Gasser AM et al. Estudo da expressão citofotométrica do marcador tumoral Caspase-3 no adenocarcinoma de cólon. ABCD Arq Bras Cir Dig. 2007; 20(4):250-3

14. Benoy I, Salgado R, Elst H, Dam P, Weyler J, Van Marck E et al. Relative microvessel área of the primary tumour, and not lymph node status, predicts the presence of bone marrow micrometatases detected by reverse transcriptase polymerase chain reaction in patients with clically non-metastatic breast cancer. Breast Cancer Res. 2005; 7:R210-R219.

15. Byrnes K, White S, Chu Q, Meschonat C, Yu H, Johnson L et al. High elF4E, VEGF, and microvessel density in stage I to III breast cancer. Ann Surg. 2006; 243(5): 684-92.

16. Monsky WL, Mouta Carreira C, Tsuzuki Y, Gohongi T, Fukumura $D$, Jain RK. Role of host microenvironment in angiogenesis and microvascular functions in human breast cancer xenografts: mammary fat pad versus cranial tumors. Clin Cancer Res. 2002; 8(4):1008-13

17. Chen T, Luo I, Mikhail N, Raskova J, Raska K. Comparison on flow and image cytometry for DNA content analysis of fresh and formalin-fixed, paraffin-embedded tissue in breast carcinoma. Cytometry. 1995; 22(3):181-9.

Recebido em 12/12/2008

Aceito para publicação em 13/02/2009

Conflito de interesse: nenhum

Fonte de financiamento:nenhuma

\section{Como citar este artigo:}

Passos FS, Cuenca RM, Malafaia O, Ribas-Filho JM, Czeczko NG, Nassif PAN, Ribas CAPM. Expressão citofotométrica quantitativa da caspase3 e do CD-34 no carcinoma ductal infiltrante de mama. Rev Col Bras Cir. [periódico na Internet] 2009; 36(5). Disponível em URL: http:// www.scielo.br/rcbc

\section{Endereço para correspondência:}

Ronaldo Máfia Cuenca

E-mail: rmcuenca@uol.com.br 